\title{
Total Quality Management (TQM) als Tool zur Hochschulentwicklung
}

\author{
Gerd Stegemann, Nicole Petschick, László Ungvári
}

\section{Allgemeiner Anspruch}

Die Technische Fachhochschule Wildau ist eine moderne, praxisverbundene, zukunftsorientierte und traditionsbewusste Hochschule. Es kann eingeschätzt werden, dass sie sich in den zurïckliegenden Jahren sowohl in Lehre und Forschung, im Technologietransfer als auch in der Verwaltungstätigkeit Dank des Engagements und der Arbeit aller Mitarbeiter erfolgreich entwickelt hat.

Gemeinsam mit Vertretern aus Wirtschaft, Verwaltung und Politik wurden in den letzten Jahren moderne und zukunftsorientierte Studiengänge konzipiert, welche an den erkennbaren zukünftigen Erfordernissen und dem Innovationsbedarf der Wirtschaft und Gesellschaft ausgerichtet sind und eine effiziente Hochschultätigkeit gestatten.

Auf dem Gebiet der Drittmittelforschung und des Technologietransfers behauptet die TFH Wildau seit Jahren Spitzenplätze unter den Fachhochschulen in der Bundesrepublik. Von großer Bedeutung dafür war das Bemuihen um Internationalisierung der Arbeit. Es gilt jedoch insbesondere auch für Hochschulen, der Erfolg von gestern zählt morgen nicht mehr.

Im Sinne des Erhalts, der weiteren Entwicklung und des Ausbaus der Wettbewerbsposition der TFH Wildau in einer national und international vernetzten Hochschullandschaft strebt sie aus diesem Grund nach Sicherung nachhaltiger Spitzenleistungen in Lehre und Studium, in der Forschung, im Technologietransfer sowie in der Verwaltung. Es zeigt sich, dass in Analogie zur Wirtschaft dynamisches und umfassendes Qualitätsmanagement auch im Hochschulwesen als ein wesentliches Tool zur Gewährleistung nachhaltigen Erfolgs durch Ausschöpfung hochschulinterner und -externer Erfolgpotenziale anzusehen ist.

Wichtig ist es, dieses Managementwerkzeug ganz präzise für die Verhältnisse und Bedingungen der TFH Wildau zu entwickeln und schöpferisch umzusetzen. Dazu werden prinzipiell drei Anwendungsfelder gesehen:

- Einbindung des TQM in das Hochschulmanagement,

- Auseinandersetzung mit externen, insbesondere staatlichen Qualitätssicherungsansprüichen sowie

- Nutzung des TQM zur Gestaltung und Profilierung der Hochschule im Sinne einer intellektuellen Reform.

Vorliegender Beitrag stellt Erkenntnisse aus der Planungs- und Analysephase des Projektes „Einrichtung und Gestaltung des TQM an der TFH Wildau“ vor.

\section{Total Quality Management als Managementtool}

Bezogen auf die Bedingungen einer Hochschule ist Total Quality Management (TQM) oder auch Umfassendes Qualitätsmanagement eine Managementmethode, welche ausgehend von den berechtigten und angemessenen Erfordernissen und Erwartungen ihrer Interessenpartner (s. Tab. 1) durch strategisch ausgerichtete Führungstätigkeit eine hohe Zufriedenheit dieser Interessenpartner und nachhaltigen Erfolg für die Hochschule anstrebt.

\begin{tabular}{|l|l|}
\hline $\begin{array}{l}\text { Interessenpartner } \\
\text { (DIN EN ISO 8004-2000) }\end{array}$ & Beispiele für Hochschulen \\
\hline Kunden und Endabnehmer & $\begin{array}{l}\text { Studierende, Wirtschaft, Behörden, } \\
\text { Forschungsauftraggeber }\end{array}$ \\
\hline Personen in der Organisation & $\begin{array}{l}\text { Hochschulmitarbeiter, Lehrbeauftragte, } \\
\text { Gastdozenten }\end{array}$ \\
\hline Eigentümer und Investoren & Gesellschaft \\
\hline Lieferanten und Partner & $\begin{array}{l}\text { Forschungspartner, Internationale Koopera- } \\
\text { tionspartner, Gymnasien, Praktikumsbetriebe, } \\
\text { Politiker, Sponsoren, Ausrüstungslieferanten }\end{array}$ \\
\hline Gesellschaft & Mitglieder der Gesellschaft \\
\hline
\end{tabular}

Tab. 1: Interessenpartner am Beispiel von Hochschulen

Abbildung 1 fasst Charakteristika des Total Quality Management zusammen und hebt das Streben nach kontinuierlicher Verbesserung (KVP) als grundlegenden Anspruch des TQM hervor.

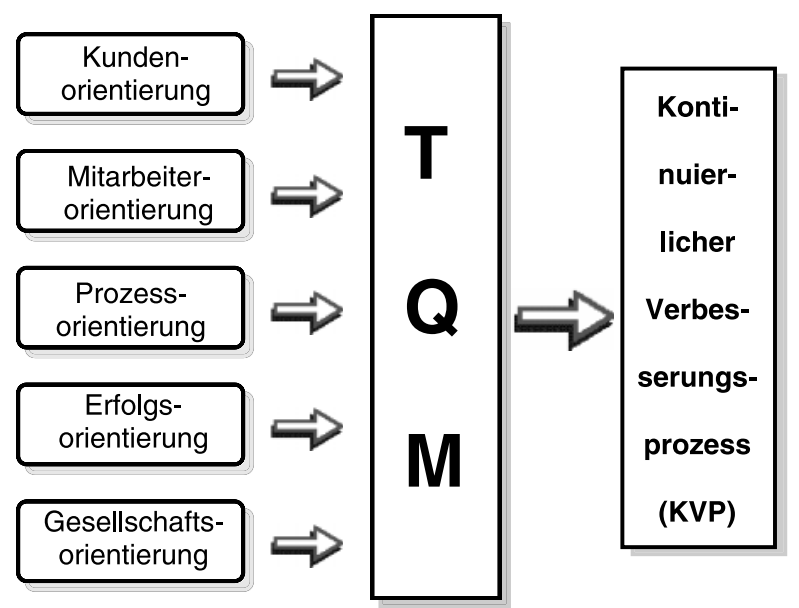

Abb. 1: Charakteristika des Total Qualität Management (TQM)

Es gilt als unbestritten, dass sich TQM sowohl in produzierenden als auch in Dienstleistungsunternehmen in den zurückliegenden Jahren im globalen Wettbewerb als ein entscheidender Faktor für die Gewährleistung nachhaltigen Geschäftserfolgs erwiesen hat. Logisch ist, dass das, was im harten Wettbewerb der Wirtschaft gilt, in anderen Bereichen des gesellschaftlichen Lebens nicht falsch sein kann. Es ist aus dem Grund kein Zufall, dass 
sich die Hochschulrektorenkonferenz (HRK) in den zurückliegenden Jahren intensiv mit Fragen der Qualitätssicherung auseinandergesetzt hat und auf den Gebieten Evaluation und Akkreditierung erkennbare Erfolge erzielt wurden.

Neben den in diesem Zusammenhang entstandenen und bewährten Qualitätssicherungsstandards bieten das weltweit genutzte QM-Regelwerk der ISO 9000:2000Normenfamilie, das EFQM-Modell für Excellenze und die internationale Norm ISO 45013 gute Ansätze für die systematische Gestaltung des TQM im Hochschulbereich (Systematisierungsgrundlagen). Selbstverständlich sind in diesem Gestaltungsprozess gesellschaftliche Forderungen, z. B. des Hochschulrahmengesetzes (HRG) oder des Brandenburgischen Hochschulgesetzes (BbgHG), umzusetzen.

Im Rahmen eines mit Jahresbeginn 2002 an der TFH Wildau gestarteten Projektes „Entwicklung und Gestaltung des TQM an der TFH Wildau“ werden nachfolgende Arbeitsschwerpunkte als besonders dringlich angesehen:

- Identifizierung der Erfolgspotenziale (Evaluation der Qualitätsarbeit) und deren planmäßige Realisierung,

- Bildung arbeitsfähiger Projektteams,

- Gewährleistung einer durchgängigen und verbindlichen Organisationsstruktur für die Fachhochschule sowie Diskussion und Bestätigung strategischer Hochschulleitlinien,

- Organisation und Realisierung aufgabenbezogener Schulungs- und Weiterbildungsaktivitäten,

- Identifikation der Interessenpartner der Fachhochschule sowie Beschreibung ihrer berechtigten Erfordernisse und Erwartungen,

- Definition des Erfolgsbegriffs für die Fachhochschule sowie Ableitung von Qualitätszielen auf der Basis von Bestwerten und deren Realisierung,

- ganzheitliche Prozessidentifikation, -analyse und gestaltung sowie -realisierung fuir Lehre und Studium, Forschung, Technologietransfer sowie Verwaltung,

- Weiterentwicklung des Hochschulcontrollings auf der Basis eines „Messsystems“ zur Qualitätslenkung und Erfolgsbewertung.

\section{Interne Evaluation der Qualitätsarbeit}

Als wesentliche Zielstellungen der internen Evaluation wurden angesehen:

- Analyse und Beschreibung des Ist-Zustandes qualitätssichernder Tätigkeiten in der Hochschule sowie

- Ermittlung von Daten als Grundlage für die Erstellung eines Projektplan-Entwurfes zur Einrichtung und Gestaltung des TQM an der TFH Wildau.

Ein so erarbeiteter Evaluationsbericht bezieht sich auf die von den Assessoren identifizierten Entwicklungspotenziale (Abweichungen) in Gegenuiberstellung von Empfehlungen/Forderungen o. a. Systematisierungsgrundlagen zum Qualitätsmanagement, zu rechtlichen Forderungen nach dem Hochschulrahmengesetz (HRG) und dem Brandenburgischen Hochschulgesetz (BbgHG).
Die Realisierung der internen Evaluation erfolgte vornehmlich in Gruppen- und Einzelgesprächen mit Mitarbeitern und Studenten der TFH Wildau. Darüber hinaus flossen in die Einschätzung Ergebnisse einer Mitarbeiterbefragung (Fragebogen der Auftaktveranstaltung zum Projekt) sowie Erkenntnisse aus der Auftaktveranstaltung selber ein. Zusätzlich erfolgte die Einsichtnahme in ausgewählte Dokumente der TFH Wildau, wie z. B. „Amtliche Mitteilungen“, Hochschulentwicklungsplanung, Imageanalyse der TFH Wildau, Haushaltsplanunterlagen, Studienfuihrer, Informationsmaterial.

Im Ergebnis der internen Evaluation kann festgestellt werden, dass die in der Hochschule gelebte Führungstätigkeit, das vorhandene, aber doch recht differenzierte Qualitätsbewusstsein ihrer Mitglieder und die Qualität von Lehre, Forschung, Technologietransfer sowie die der „internen Dienstleistungen“ solide Voraussetzungen für die Entwicklung und Realisierung eines anspruchsvollen TQM-Konzeptes darstellen. Ungeachtet dessen konnte bei der Evaluierung eine erhebliche Anzahl nicht gleichgewichtiger Potenziale zur Optimierung von Geschäftsprozessen ermittelt werden. Unter Berücksichtigung der Tatsache, dass eine intensive interne und externe Kommunikation als kritischer Erfolgsfaktor anzusehen sind, ergeben sich wesentliche Charakteristika fuir die Projektrealisierung, wie:

- die Gründung der Arbeiten auf den vorhandenen Stärken,

- ein modernes Selbstverständnis der TFH,

- eine hohe Mitarbeiter-, Kunden- und Prozessorientierung,

- die Anerkennung und Realisierung des Prinzips kontinuierlicher Verbesserungen sowie

- die Entwicklung der Hochschule zu einer lernenden Organisation.

Als Schwerpunkt für den erfolgreichen Projektanlauf in der Realisierungsphase wird die umfassende Wahrnehmung der Führungsverantwortung angesehen, wozu u. a. gehören:

- aufbauend auf Visionen zur Hochschulentwicklung Hochschulleitlinien abzuleiten und diese auf der Grundlage einer bestätigten Qualitätspolitik durch intensive interne Kommunikation für die tägliche Arbeit zu erschließen,

- die Definition eines hierarchisch gegliederten Erfolgsbegriffes sowie

- die Schaffung von Rahmenbedingungen für eindeutige Zuständigkeitsregelungen.

\section{Qualitätsverbesserungsprogramm}

Basis für die Erarbeitung eines Qualitätsverbesserungsprogramms und dessen Realisierung bilden die im Evaluationsbericht erfassten Entwicklungspotenziale und die diesen zugeordneten Regelungsempfehlungen (s. Abb. 2).

Eine zentrale Rolle in diesem Prozess nimmt der Lenkungsausschuss zum Projekt ein. Dem Lenkungsaus- 


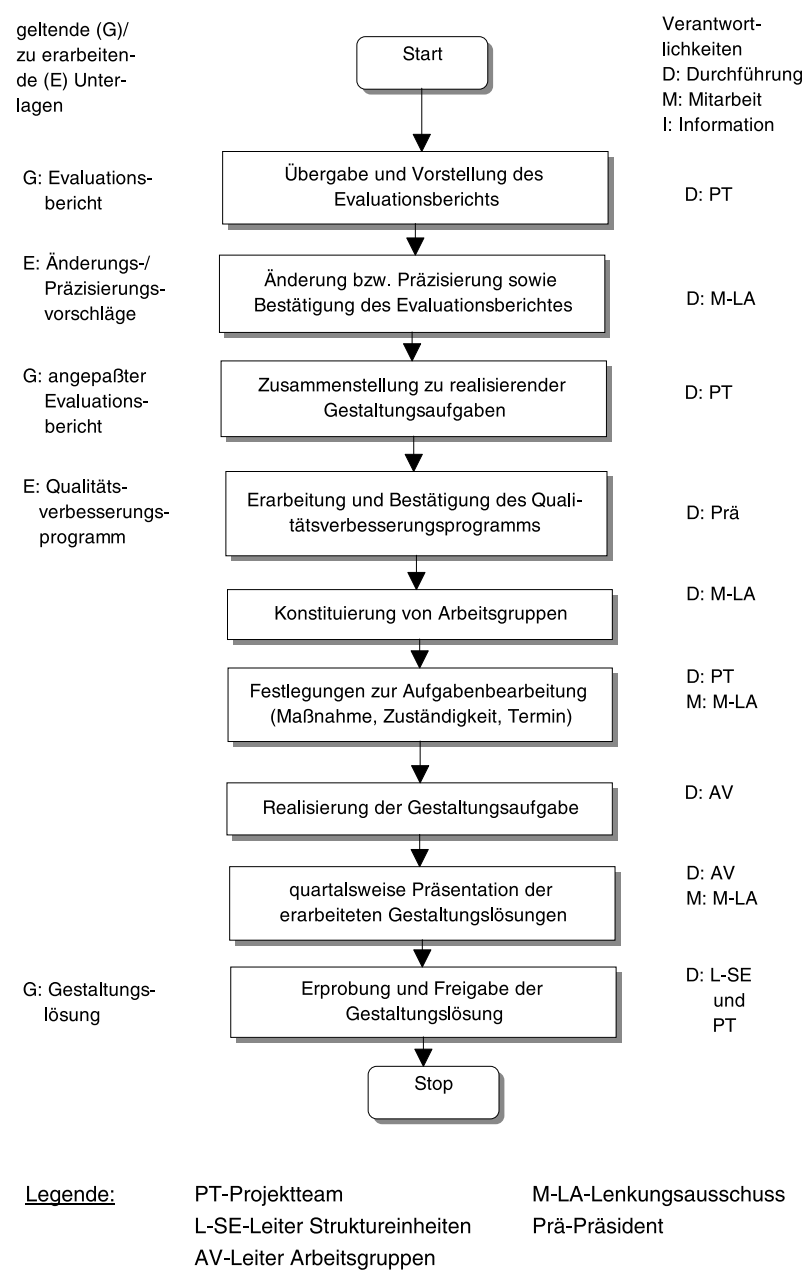

Abb. 2: Ablaufalgorithmus für die Erarbeitung des Qualitätsverbesserungsprogramms und dessen Realisierung

schuss gehören an: der Präsident, die Vizepräsidentin/ der Vizepräsident, die Kanzlerin, die Dekane, die Vorsitzenden des Akademischen Senats, des Personal- und Studentenrates, die Gleichstellungs- und die Qualitätsbeauftragte sowie der hochschulexterne Moderator. Im Auftrag des Präsidenten koordiniert und organisiert er die Projekttätigkeiten, initiiert Arbeitsgruppen und er-

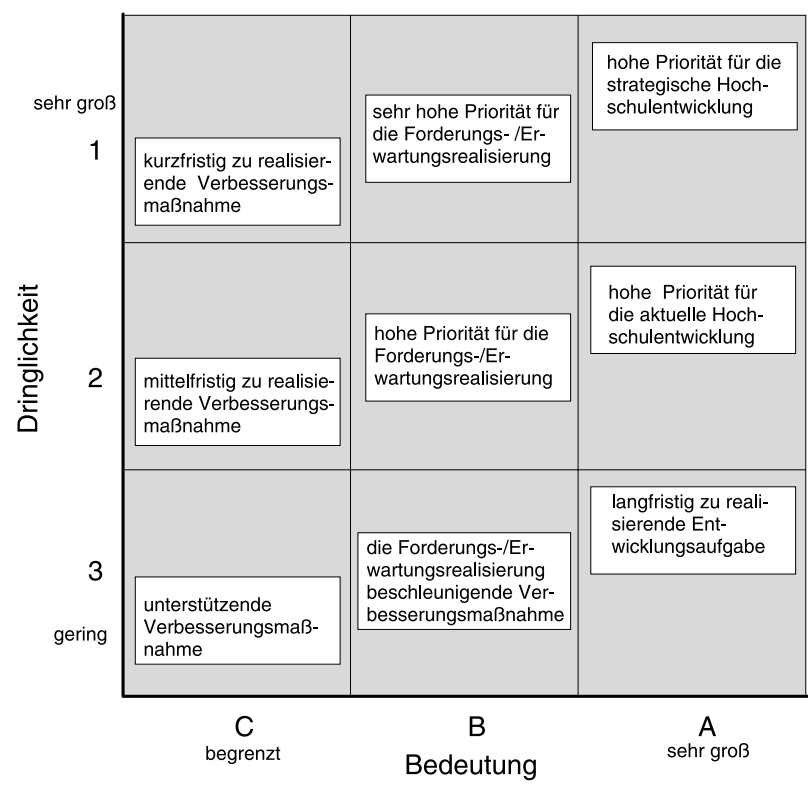

Abb. 3: Portfolio zur Prioritätenbildung von Verbesserungsmaßnahmen arbeitet Entscheidungsempfehlungen für den Präsidenten. $\mathrm{Zu}$ seinen wichtigen Aufgaben gehört die Auswahl zu realisierender Gestaltungsaufgaben, deren Prioritätenbildung, Terminierung sowie die abschließende Bewertung der Gestaltungslösungen (siehe Abb. 2). Grundlage der Prioritätenbildung sind die inhaltlich strategische Bedeutung der Einzelmaßnahme sowie ihre terminliche Dringlichkeit. Dazu erforderliche Entscheidungen stuitzen sich auf Abbildung 3.

Im Ergebnis dieser Tätigkeit entstand ein hochschulspezifisches Qualitätsverbesserungsprogramm mit mehr als 40 differenzierten Aufgabenstellungen (s. www.tfhwildau.de/proqm/qmandertfh.htm). Beispiele aus dem Qualitätsverbesserungsprogramm enthält Tabelle 2.

\begin{tabular}{|l|l|}
\hline Kategorie & Verbesserungsmaßnahme (Beispiel) \\
\hline A 1 & $\begin{array}{l}\text { Entwicklung und Realisierung strategisch ausgerichteter Hoch- } \\
\text { schulleitlinien }\end{array}$ \\
\hline A 2 & $\begin{array}{l}\text { Erarbeitung und Realisierung eines Konzeptes zum Informations- } \\
\text { management des Immatrikulations- und Prüfungsamtes }\end{array}$ \\
\hline A 3 & $\begin{array}{l}\text { Erarbeitung und schrittweise Realisierung eines Konzeptes „TFH } \\
\text { Wildau als lernende Organisation“ }\end{array}$ \\
\hline B 1 & $\begin{array}{l}\text { Entwicklung und Realisierung eines Schulungs- und Weiter- } \\
\text { bildungsprogramms }\end{array}$ \\
\hline B 2 & $\begin{array}{l}\text { Entwicklung und Realisierung eines Leitfadens „Entwicklung von } \\
\text { Studiengängen“ }\end{array}$ \\
\hline B 3 & $\begin{array}{l}\text { Erarbeitung eines Konzeptes für die systematische Begabtenför- } \\
\text { derung }\end{array}$ \\
\hline C 1 & $\begin{array}{l}\text { Durchführung einer Ist-Zustandsanalyse zur Publikationstätigkeit } \\
\text { an der TFH Wildau }\end{array}$ \\
\hline C 2 & keine Maßnahme \\
\hline C 3 & $\begin{array}{l}\text { Erarbeitung eines Leitfadens „Vor- und Nachbereitung externer } \\
\text { Evaluationen von Studienfächern“ }\end{array}$ \\
\hline
\end{tabular}

Tab. 2: Nach Kategorien gegliederte Beispiele des Qualitätsverbesserungsprogramms

Durch die Bestätigung des Qualitätsverbesserungsprogramms durch den Präsidenten wird es zum offiziellen Arbeitsmaterial der Fachhochschule und steht ihren Mitarbeitern für eine aktive Beteiligung bei der Realisierung zur Verfuigung. Es soll sie zur freiwilligen Mitwirkung in Arbeitsgruppen, Qualitätsverbesserungsteams, Qualitätszirkeln sowie zur Teilnahme an Schulungs- und Weiterbildungsmaßnahmen mit dem ausdruicklichen Ziel anregen, immer stärker von „Problemdiskussionen“ zur Lösung zukunftsorientierter Aufgabenstellungen zu kommen. So soll die Mitarbeiterakzeptanz für notwendige Veränderungen in der Hochschule gefördert werden. Als besonders wichtig wird dafür eine kontinuierliche, angemessene und verständliche Darstellung der Ziele, Aufgaben und Ergebnisse der Projektarbeit angesehen.

Den Vorschlag für zu bildende Arbeitsgruppen enthält Tabelle 3.

\begin{tabular}{|l|l|}
\hline Arbeitsgruppe & Unterarbeitsgruppe \\
\hline Strategieentwicklung & - \\
\hline Prozessgestaltung & $\begin{array}{l}\text { Prozesskoordinierung } \\
\text { Lehre und Studium } \\
\text { Forschung und Technologietransfer } \\
\text { Interne Dienstleistungen } \\
\text { Hochschulmarketing }\end{array}$ \\
\hline Lernende Organisation & - \\
\hline Information/Kommunikation & \\
\hline Controlling & - \\
\hline QM-System & - \\
\hline
\end{tabular}

Tab. 3: Arbeitsgruppen und deren Untersetzung zum Projekt 


\section{Prozessorientierung}

Im Sinne der Prozessorientierung waren im Rahmen der internen Evaluation zunächst die Geschäftsprozesse der TFH Wildau zu identifizieren. In Tabelle 4 sind die nach Kern-, Management- und Unterstuitzungsprozessen gegliederten Geschäftsprozesse zusammengefasst. Prinzipiell liegen dieser Klassifizierung nachfolgende Arbeitsdefinitionen zu Grunde:

\section{- Kernprozess}

„Prozess, dessen Aktivitäten direkten Bezug zur Lehre, Forschung und zum Technologietransfer aufweisen und damit einen Beitrag zur Erfüllung der berechtigten und angemessenen Erwartungen und Forderungen der Interessenpartner der Fachhochschule leisten."

\section{- Managementprozess}

„Prozess, dessen Aktivitäten durch Regelungs- und Entscheidungscharakter zur Ableitung und/oder Sicherstellung der Hochschulstrategie und -ziele beitragen."

\section{- Unterstützungsprozess}

„Prozess, dessen Aktivitäten nicht selbst zur Realisierung der berechtigten und angemessenen Erwartungen und Forderungen der Interessenpartner und/oder zur Entscheidungsfindung erforderlich sind, aber notwendig sind, um Kern- und Managementprozesse ausfuihren zu können.“

Aufgabe der Unterarbeitsgruppe „Prozesskoordinierung“ ist es, die Entwicklung einer auf eine effiziente und optimale, ergebnisorientierte Prozessrealisierung gerichtete Input-/Outputvernetzung zu initiieren (s. Abb. 4).

Von ganz besonderer Bedeutung für die Qualitätslenkung ist in dem Zusammenhang die Entwicklung eines Messsystems zur Erfassung und Bewertung der Wirkungen von In- und Outputs.

Basis einer komplexen Prozessbetrachtung und -gestaltung ist eine umfassende Prozessanalyse unter weitgehender Einbeziehung der Prozesseigner.

Angestrebt wird, die identifizierten Prozesse mit dem Ziel einer angemessenen Erfuillung der berechtigten Erwartungen und Forderungen der Interessenpartner der TFH Wildau in ihrer Gesamtheit weiter zu vervollkomm-

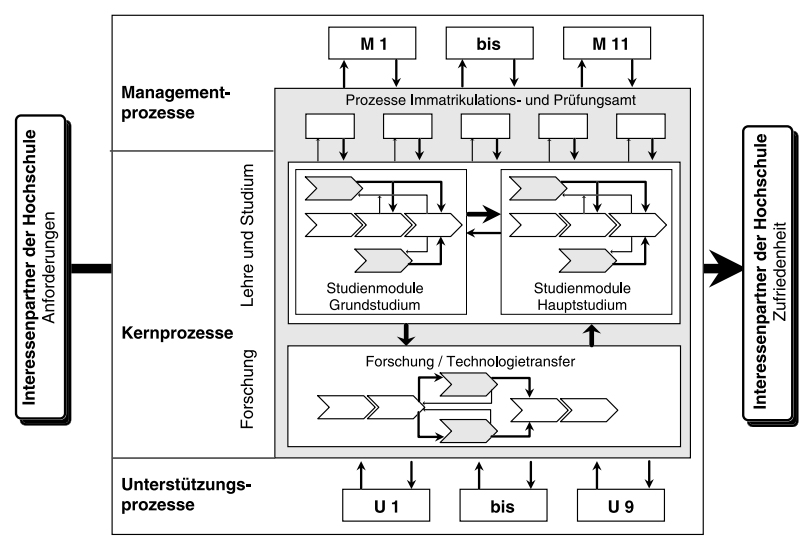

Abb. 4: Allgemeine Prozessgliederung und deren Input-/Outputvernetzung

nen, verbindlich festzulegen und zu dokumentieren. Dabei geht es nicht nur um die Optimierung organisatorischer und logistischer Abläufe, sondern vor allem auch um eine intellektuelle Reform. Gleichzeitig muss die Bewertung von Messgrößen systematische Ansatzpunkte fuir den kontinuierlichen Verbesserungsprozess bereitstellen. Basis eines hochschulspezifischen Messsystems sollten sowohl monetäre als auch nicht monetäre Indikatoren, z. B. auf der Grundlage des Balanced Scorecard-Ansatzes sein (Abb. 5).

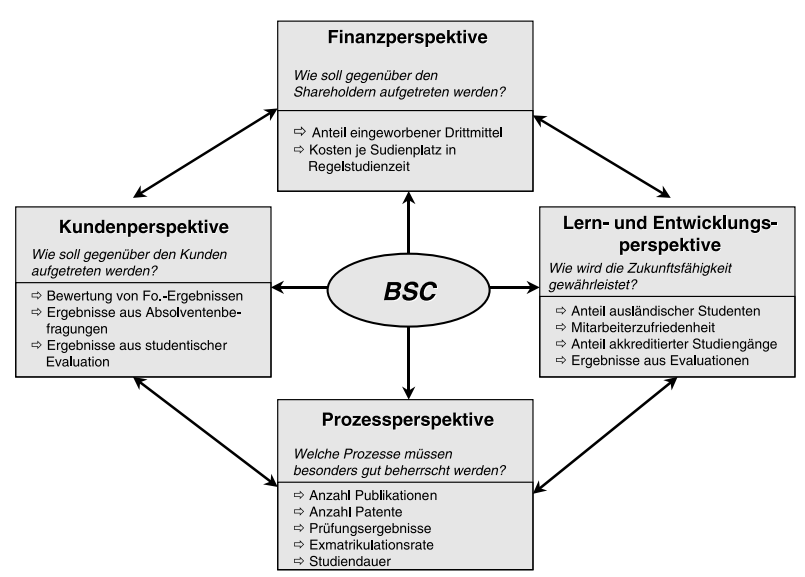

Abb. 5: Perspektiven des Balanced-Scorecard-Ansatzes mit Beispielkenngrößen

Grundsätzlich dürfen die Möglichkeiten selbst einer exzellent realisierten Prozessoptimierung nicht überschätzt werden. So sind z. B. tiefe Einschnitte in der Ressourcenbereitstellung und eine nachhaltige Verbesserung der Qualität in Lehre und Forschung miteinander nicht vereinbar.

\begin{tabular}{|c|c|c|}
\hline Kernprozesse & Managementprozesse & Unterstützungsprozesse \\
\hline $\begin{array}{l}\text { K } 1 \text { Entwicklung von Studiengängen } \\
\text { K } 2 \text { Studienberatung und Akquisition } \\
\text { K } 3 \text { Lehre } \\
\text { K } 4 \text { Begabtenförderung } \\
\text { K } 5 \text { Praktikumsregelung und -bewertung } \\
\text { K } 6 \text { Prüfung } \\
\text { K } 7 \text { Internationale Kooperation } \\
\text { K } 8 \text { Drittmittelforschung und Technologietransfer } \\
\text { K } 9 \text { Publikationstätigkeit }\end{array}$ & $\begin{array}{ll}\text { M } 1 & \text { Strategische Hochschulentwicklung } \\
\text { M } 2 & \text { Personalmanagement } \\
\text { M } 3 & \text { Partnerschaftsmanagement } \\
\text { M } 4 & \text { Benchmarking } \\
\text { M } 5 & \text { Hochschulmarketing } \\
\text { M } 6 & \text { Internes Dienstleistungsmanagement } \\
\text { M } 7 & \text { Im- und Exmatrikulation } \\
\text { M } 8 & \text { Interne Evaluation } \\
\text { M } 9 & \text { Hochschulcontrolling } \\
\text { M } 10 & \text { Fehler-, Beschwerde- und Verbesserungs- } \\
& \text { management } \\
\text { M } 11 \text { Risikomanagement }\end{array}$ & $\begin{array}{l}\text { U } 1 \text { Prozessmanagement } \\
\text { U } 2 \text { Informations- und Kommunikationsmanagement } \\
\text { U } 3 \text { Beschaffung } \\
\text { U } 4 \text { Ressourcenmanagement } \\
\text { U } 5 \text { Rechnungswesen } \\
\text { U } 6 \text { hochschulinterne Aufgaben der externen Evaluation } \\
\text { und Akkreditierung } \\
\text { U } 7 \text { Betrieb und Erhaltung } \\
\text { U } 8 \text { Dokumentationsmanagement } \\
\text { U } 9 \text { Qualitäts-, Umwelt- und Sicherheitsmanagement }\end{array}$ \\
\hline
\end{tabular}

Tab. 4: Prozesszusammenstellung 


\section{Dokumentationsmanagement}

\subsection{Lenkung der Dokumente}

An der TFH Wildau existieren eine Reihe von Dokumenten, z. B. Amtliche Mitteilungen, Rundschreiben, Protokolle, Formulare und Berichte mit Weisungs- oder Richtliniencharakter sowie Nachweisdokumente, wie z. B. Prüfungs- und Messprotokolle (Abb. 6).

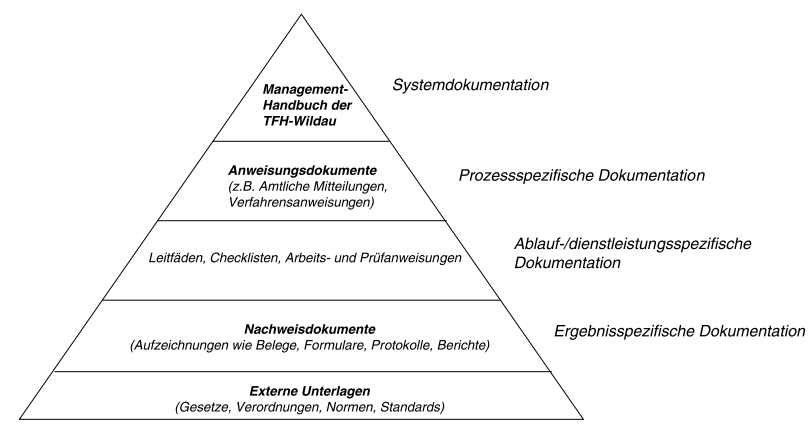

Abb. 6: Vorschlag für eine hierarchisch gegliederte Dokumentenstruktur

Für die Gestaltung des Dokumentationsmanagements wurden in der internen Evaluation wesentliche Entwicklungspotenziale identifiziert:

- die Abgrenzung und Beschreibung der Bedingungen für das Dokumentenformat (Papier, elektronisch),

- die Festlegung eines einheitlichen Layouts,

- die Erstellung eines elektronischen Musterordners, im welchem Vorlagen, z. B. für Berichte, Protokolle, enthalten sind,

- die Festlegung einer Gliederungssystematik, um Dokumente eindeutig zu kennzeichnen und zuzuordnen,

- die Festlegung der Zuständigkeiten für das Handling (erarbeiten, prüfen, freigeben, ändern, archivieren) von Dokumenten und externer Unterlagen sowie

- der Festlegung eines Verteilersystems entsprechend den Dokumentenarten.

\subsection{Elektronisches Dokumentenmanagement}

An der TFH Wildau werden Dokumente zum größten Teil in Papierform erstellt und verteilt. Wesentliche Nachteile werden durch die Autoren insbesondere in den hohen Aufwendungen (u. a. Zeit, Papier) für die Erstellung, Verteilung, Aktualisierung von Dokumenten und in dem nicht unerheblichen Zeitversatz zwischen der Fertigstellung der Dokumente und deren Wahrnehmung durch den Nutzer gesehen.

Im Sinne einer wirksamen und effizienten Information und Kommunikation an der TFH Wildau ist dem elektronischen Dokumentenmanagement ein höherer Stellenwert beizumessen. Wichtige Vorteile des elektronischen Dokumentenmanagements sind $\mathrm{z}$. B.:

- Dokumente liegen an einer zentralen Stelle,

- Änderung bzw. Austausch von Dokumenten erfolgt lediglich einmal,

- Verteilung/Freigabe von Dokumenten erfolgt schnell; alle vernetzten Nutzer sind sofort erreichbar,

- Übersichtlichkeit uiber freigegebene Dokumente gegeben,
- nur der aktuelle Stand der Dokumente liegt an der zentralen Stelle,

- kein Verlust von Dokumenten.

Für die Einrichtung eines elektronischen Dokumentenmanagements gibt es in der Praxis verschiedene Lösungen. In den nachfolgenden Ausfuihrungen wird eine mögliche Variante vorgestellt.

Die Dokumente werden mittels Softwareprogrammen (z. B. Word, Excel) durch den Zuständigen erstellt und nach Prüfung auf fachliche Richtigkeit, Vollständigkeit, Angemessenheit in ein PDF-Format umgewandelt. Die PDF-Dateien werden auf einer zentralen Stelle (FTPSERVER) abgelegt und können durch den Nutzer entsprechend den festgelegten Zugriffsrechten abgerufen werden.

Durch die Umwandlung der Dokumente in ein PDF-Format sind nachfolgende Kriterien gewährleistet:

- Schutz von Dokumenten vor unberechtigter Handhabung (Zugriffs-, Kopier-, Druck-, Bearbeitungsschutz),

- Verknuipfungen innerhalb und zwischen Dokumenten,

- schnelles und sicheres Bewegen im Dokument durch Navigationsfunktionen (Lesezeichen),

- geringe Lade- und Übertragungszeiten durch kleine Dateigrößen,

- Übereinstimmung der Bildschirmausgabe mit der Druckausgabe,

- Lese-, Drucksoftware (Acrobat-Reader) kostenlos,

- Druck von benötigten Papierdokumenten (z. B. Formulare) vor Ort.

\section{Zusammenfassung}

Im vorliegenden Beitrag werden Erkenntnisse aus der Planungs- und Analysephase des Projektes „Einrichtung und Gestaltung des TQM an der TFH Wildau“ beschrieben. Ausgehend vom allgemeinen Anliegen dieses Projektes werden die Vorbereitung und Realisierung einer internen Evaluierung zur Ist-Zustandsermittlung des Niveaus der Qualitätsarbeit an der TFH Wildau und die Nutzung dabei erzielter Ergebnisse vorgestellt.

Aspekte der Prozessorientierung und des Dokumentenmanagements bilden eine wesentliche Basis für die konstruktive Umsetzung der Ziele des Projektes.

Als Resümee lässt sich feststellen, dass die in der Hochschule gelebte Führungstätigkeit, das vorhandene, aber doch recht differenzierte Qualitätsbewusstsein ihrer Mitglieder und die Qualität von Lehre, Forschung, Technologietransfer sowie die der ,internen Dienstleistungen" solide Voraussetzungen für die Entwicklung und Realisierung eines anspruchsvollen TQM-Konzeptes darstellen. Ungeachtet dessen bestehen jedoch erhebliche Entwicklungspotenziale, welche nach Prioritätenbildung im Rahmen von Verbesserungsmaßnahmen erschlossen werden sollten. 


\section{Autoren}

Prof. Dr.-Ing. habil Gerd Stegemann

Tel. (0 33 75) 508-914

E-Mail: qmstegem@igw.tfh-wildau.de

Dipl.-Betriebswirtin (FH) Nicole Petschick

Technische Fachhochschule Wildau

Fachbereich Ingenieurwesen/Wirtschaftsingenieurwesen

Tel. (0 33 75) 508-915

E-Mail: qmpetsch@igw.tfh-wildau.de

Prof. Dr. László Ungvári (Präsident)

Technische Fachhochschule Wildau

Tel. (0 33 75) 508-100

E-Mail: ungvari@wi-bw.tfh-wildau.de 